\title{
Neutrino decay to electron and $W$ boson in a superstrong magnetic field in the Early Universe
}

\author{
Alexander Kuznetsov ${ }^{1, \star}$, Alexander Okrugin ${ }^{1, \star \star}$, and Anastasiya Shitova ${ }^{1, \star \star \star}$ \\ ${ }^{1}$ Division of Theoretical Physics, Department of Physics, Yaroslavl State P. G. Demidov University, \\ Sovietskaya 14, 150000 Yaroslavl, Russian Federation
}

\begin{abstract}
An intense electromagnetic field makes possible the process of the neutrino decay into electron and $W$ boson that is kinematically forbidden in a vacuum. The exact formula for the probability of ultra high energy neutrino decay in a superstrong magnetic field via the representation for the propagators of the charged particles expanded as sums over Landau levels is derived. The obtained function clarifies previously known approximate formula. The neutrino mean free path is estimated. It is shown, that the resonance peaks present in the decay width for certain neutrino energies correspond to the electron and the $W$ boson creation at specific Landau levels. Some interesting possible manifestations of the process in the conditions of the Early Universe are briefly discussed.
\end{abstract}

\section{Introduction}

In recent decades, the studies of electroweak processes in active media (such as plasma or strong magnetic fields) became noticeably popular. In many respects it is connected with rapidly developing concept of magnetars, i.e. neutron stars which evolution is driven largely by magnetic fields [1]. Other examples when a strong magnetic field could possibly manifest itself are the conditions in the Early Universe, and the experiments at modern colliders, e.g. non-central collisions of protons and heavy ions.

Extreme physical conditions actively influence the run of quantum processes, thus allowing or enhancing the transitions that are forbidden or strongly suppressed in vacuum. The external field effects should be taken into account on the basis of exact solutions of the field theory equations for a charged particle in an external electromagnetic field, but not on the basis of perturbation theory.

The strength of the fields we discuss is essentially greater than the so-called Schwinger limit $B_{e}=$ $m_{e}^{2} / e \simeq 4.4 \times 10^{13} \mathrm{G}$, which is the naturally arising scale for the field strength as the quantizing field for an electron. Hereafter, we use the Planck units: $\hbar=1, c=1$, and the Minkowski metric with the signature $(+---)$. Another limit that can be naturally introduced is defined by the mass of the gauge boson $m_{W}$ and can serve as a boundary of applicability of Standard Model: $B_{W}=m_{W}^{2} / e \simeq 1.1 \times 10^{24} \mathrm{G}$. There arises a question whether the electroweak vacuum remains stable at $B \rightarrow B_{W}$. As it was shown in Ref. [2], the radiation corrections act to prevent the instability of the electroweak vacuum in strong fields.

\footnotetext{
^e-mail: avkuzn@uniyar.ac.ru

$\star \star$ e-mail: okrugin@uniyar.ac.ru

$\star \star \star$ e-mail: pick@mail.ru
} 
Magnetic fields exist at all the scales of the Universe structure: from planets to galaxy clusters. It is used to think that magnetic fields arised yet in the Early Universe, but until now it is not fully understood what is the preferential mechanism of formation of contemporary observable magnetic fields. There are plenty of ideas that can explain the evolution of some seed field to the modern scales $[3,4]$. Primordial magnetic fields are worth to study not only for the reason to explain the observable fields but also because they open a possibility to study fundamentally new physics at the stage of the Early Universe.

A problem of studying possible influence of an active environment on the neutrino dispersion properties is quite important. As it was already mentioned, the intense magnetic field opens new possibilities for the processes that are kinematically forbidden in a vacuum. Probably, the most interesting processes of such kind are the transitions of a massless neutrino: the production of a lepton pair by the neutrino, $v \rightarrow v \ell^{-} \ell^{+}$, and the neutrino decay into $W^{+}$boson and a charged lepton, $v_{\ell} \rightarrow \ell^{-} W^{+}$ $(\ell=e, \mu, \tau)$ in a strong field. The external field influence on the propagation of charged particles is defined by it's specific charge, so, the lighter is a particle, the more sensitive it is. Thus, for the definiteness we consider an electron as the charged lepton, and the results can be generalized for the $\mu$ and the $\tau$ leptons straightforwardly. For the described conditions, only neutrinos with ultra high energy can play the role.

The calculation of the width of the process $v_{e} \rightarrow e^{-} W^{+}$in an external magnetic field has a long history. Ref. [5] was probably the first publication on the problem. A detailed analysis of this process was also performed in Ref. [6]. Later on, another authors [7] remade the calculations and came to the contradictions with the previously obtained results. To the end, in Ref. [8] the result of Ref. [6] was confirmed. The most possible reason for the discrepancy between the results was as follows: the authors of Ref. [7] used the $W$ boson propagator expanded over the field tensor $F^{\mu v}$ to the linear terms, while the quadratic terms were also essential.

Later, the process was also analysed in Ref. [9], where an expression for the decay width was obtained which was valid for an arbitrary relationship between the masses of the neutrino, charged lepton, and $W$ boson, and in the case of a left-polarized standard neutrino, the result of Ref. [8] was reproduced exactly. In Ref. [10], on the basis of an instanton model, the author also generally confirmed the results of Ref. [8].

In the present paper, we discuss extremely strong magnetic fields $\sim B_{W}$ which could be quite important while studying the processes in the conditions of the Early Universe where the fields of that strength could probably exist [11-13].

\section{Probability of the process $v_{e} \rightarrow e^{-} W^{+}$in a strong external magnetic field}

While solving series of various problems on charged particles interactions in external magnetic fields a great influence gained the method in which the contribution of the external field is considered not within the perturbation theory but on the basis of exact solutions of wave equations in external magnetic field. This method was firstly proposed by Sokolov and lately developed by Ternov while solving the problem of synchrotron radiation. The corresponding idea is now known as the Furry picture. For calculations of physical processes, the Feynman technique is used, the particles in the initial and final states are described by the wave equation for the particles in external field, and the internal lines are described by the propagators built on a base of those solutions. A more detailed description of this approach can be found e.g. in Refs. [14-19]. The method could be successfully applied in a case of strong magnetic fields when it becomes impossible to make calculations on the basis of perturbation theory. Taking into account a vacuum stability in superstrong magnetic fields, one may study processes for the values of $B$ much greater than $B_{e}$. 
The probability of the neutrino decay in an external magnetic field can be expressed via the neutrino self energy operator. The probability could be described in terms of the imaginary part of the invariant amplitude of the $v_{e} \rightarrow v_{e}$ transition via the following relation:

$$
w\left(v_{e} \rightarrow e^{-} W^{+}\right)=\frac{1}{E} \operatorname{Im} \mathcal{M}\left(v_{e} \rightarrow v_{e}\right)=-\frac{1}{E} \operatorname{Im}\left\{\operatorname{Tr}\left[\Sigma(p) \gamma_{L}(p \gamma)\right]\right\},
$$

where we took into account the expression for the density matrix of left-handed neutrinos, $\rho(p)=$ $\gamma_{L}(p \gamma) ; p^{\alpha}=(E, \mathbf{p})$ is the neutrino 4-momentum, $\gamma_{L}=\left(1-\gamma_{5}\right) / 2$.

Neutrino self energy operator is defined by the relation:

$$
\Sigma(p)=-\frac{\mathrm{i} g^{2}}{2}\left[\gamma^{\alpha} \gamma_{L} J_{\alpha \beta}^{W}(p) \gamma^{\beta} \gamma_{L}\right]
$$

The function $J_{\alpha \beta}^{W}(p)$ in Eq. (2) is an integral of the Fourier transforms of the translational-invariant parts of the electron and the $W$ boson propagators, for details see e.g. Ref. [19]:

$$
J_{\alpha \beta}^{W}(p)=\int \frac{\mathrm{d}^{4} q}{(2 \pi)^{4}} S(q) G_{\beta \alpha}^{W}(q-p)
$$
form:

The electron propagator expanded over Landau levels (see Ref. [20] and also Ref. [21]) takes the

$$
S(q)=\sum_{n=0}^{\infty} \frac{\mathrm{i}}{q_{\|}^{2}-m_{e}^{2}-2 n \beta+\mathrm{i} \epsilon}\left\{\left[(q \gamma)_{\|}+m_{e}\right]\left[d_{n}(\alpha)-\frac{\mathrm{i}}{2}(\gamma \varphi \gamma) d_{n}^{\prime}(\alpha)\right]-(q \gamma)_{\perp} 2 n \frac{d_{n}(\alpha)}{\alpha}\right\},
$$

where $\beta=e B, \alpha=q_{\perp}^{2} / \beta$, the tensor indices for 4-vectors and tensors in parentheses are assumed to be contracted consecutively, for instance: $(\gamma \varphi \gamma)=\gamma^{\alpha} \varphi_{\alpha \beta} \gamma^{\beta}$, and the following notations are used: $(q \gamma)_{\|}=(q \tilde{\varphi} \tilde{\varphi} \gamma),(q \gamma)_{\perp}=(q \varphi \varphi \gamma)$; the tensor $\varphi_{\alpha \beta}=F_{\alpha \beta} / B$ is the dimensionless electromagnetic tensor, $\tilde{\varphi}_{\alpha \beta}=\frac{1}{2} \varepsilon_{\alpha \beta \mu \nu} \varphi^{\mu v}$ is the dual dimensionless tensor $\left(\varepsilon^{0123}=-\varepsilon_{0123}=+1\right)$. In the frame where the third space axis is directed along the vector of an external magnetic field $\mathbf{B}$, the 4-vectors with the indexes $\perp$ and $\|$ belong to the Euclidean $\{1,2\}$-subspace and the pseudo-Euclidean $\{0,3\}$-subspace respectively. Then, $(\varphi \varphi)_{\alpha \beta}=\operatorname{diag}(0,1,1,0),(\tilde{\varphi} \tilde{\varphi})_{\alpha \beta}=\operatorname{diag}(1,0,0,-1)$, and $p_{\perp}=\left(0, p_{1}, p_{2}, 0\right), p_{\|}=$ $\left(p_{0}, 0,0, p_{3}\right)$. The functions $d_{n}(\alpha)$ in Eq. (4) are expressed via the Laguerre polynomials:

$$
d_{n}(\alpha)=(-1)^{n} \mathrm{e}^{-\alpha}\left[L_{n}(2 \alpha)-L_{n-1}(2 \alpha)\right],
$$

with a completion of $L_{-1}(x) \equiv 0$. In particular, for $n=0$, that is for the ground Landau level, one has:

$$
S^{(0)}(q)=\mathrm{i} \frac{(q \gamma)_{\|}+m_{e}}{q_{\|}^{2}-m_{e}^{2}+\mathrm{i} \epsilon} \mathrm{e}^{-\alpha}\left(1+\frac{\mathrm{i}}{2}(\gamma \varphi \gamma)\right) .
$$

For the $W$ boson propagator, one can find the similar expression as the sum over the Landau levels $[19,22]$ (here, we use the 't Hooft-Feynman gauge):

$$
\begin{aligned}
G_{\mu \nu}(q-p) & =\sum_{k=0}^{\infty} \frac{\mathrm{i}(-1)^{k} \mathrm{e}^{-\alpha^{\prime}}}{q_{\|}^{2}-m_{W}^{2}-\beta(2 k-1)+\mathrm{i} \epsilon}\left\{2(\tilde{\varphi} \tilde{\varphi})_{\mu \nu} L_{k-1}\left(2 \alpha^{\prime}\right)\right. \\
& \left.+(\varphi \varphi)_{\mu \nu}\left[L_{k}\left(2 \alpha^{\prime}\right)+L_{k-2}\left(2 \alpha^{\prime}\right)\right]-\mathrm{i} \varphi_{\mu \nu}\left[L_{k}\left(2 \alpha^{\prime}\right)-L_{k-2}\left(2 \alpha^{\prime}\right)\right]\right\},
\end{aligned}
$$

where $\alpha^{\prime} \equiv(q-p)_{\perp}^{2} / \beta$. 
Unlike the electron propagator, the contribution from the ground level, $k=0$, to the $W$ boson propagator has a peculiarity:

$$
G_{\mu \nu}^{(0)}(q)=\frac{\mathrm{i}}{q_{\|}^{2}-m_{W}^{2}+\beta+\mathrm{i} \epsilon} \mathrm{e}^{-q_{\perp}^{2} / \beta}\left[(\varphi \varphi)_{\mu \nu}-\mathrm{i} \varphi_{\mu \nu}\right],
$$

and it is essential that it contains a pole at $q_{\|}^{2}=m_{W}^{2}-\beta$. So, if the magnetic field rises up to the critical value for the $W$ boson, $B_{W} \simeq 1.1 \times 10^{24} \mathrm{G}$, then the already mentioned question of stability arises of the perturbation theory for the $W$ boson vacuum $[2,12]$. Gathering Eqs. (7), (4), (3) and (2) consistently, one can obtain the basic formula for the probability of the process $v_{e} \rightarrow e^{-} W^{+}$. Further integration over the 4-momentum $q$ in Eq. (3) can be separated to independent integrals over the transverse momentum $q_{\perp}$ and the longitudinal momentum $q_{\|}$.

The integrals over the transverse momentum are of the scalar and the vector type:

$$
\begin{aligned}
\mathcal{I}_{\perp} & =\int \frac{\mathrm{d}^{2} q_{\perp}}{(2 \pi)^{2}} \mathrm{e}^{-\alpha-\alpha^{\prime}} L_{n}(2 \alpha) L_{k}\left(2 \alpha^{\prime}\right)=\frac{\beta}{8 \pi} \mathrm{e}^{-\chi} L_{n}^{k-n}(\chi) L_{k}^{n-k}(\chi), \\
\mathcal{I}_{\perp}^{\mu} & =\int \frac{\mathrm{d}^{2} q_{\perp}}{(2 \pi)^{2}} \mathrm{e}^{-\alpha-\alpha^{\prime}} \frac{4 n}{\alpha} q_{\perp}^{\mu}\left(L_{n}(2 \alpha)-L_{n-1}(2 \alpha)\right) L_{k-1}\left(2 \alpha^{\prime}\right) \\
& =-\frac{\beta}{2 \pi} p_{\perp}^{\mu} \mathrm{e}^{-\chi} L_{n-1}^{k-n}(\chi) L_{k-1}^{n-k+1}(\chi),
\end{aligned}
$$

where $\chi \equiv p_{\perp}^{2} /(2 \beta)$. Note that the associated Laguerre polynomials should be additionally determined so that $L_{n}^{S}(x) \equiv 0$ in the case when $n$ is negative. The integral Eq. (9) appears also in the calculation of the fermion density matrix in a constant uniform field [23].

The integral over the longitudinal momentum $q_{\|}$has the form:

$$
\begin{aligned}
\mathcal{I}_{\|}^{\mu} & =\operatorname{Im}\left\{i \int \frac{\mathrm{d}^{2} q_{\|}}{(2 \pi)^{2}} \frac{q_{\|}^{\mu}}{\left(q_{\|}^{2}-2 n \beta\right)\left((q-p)_{\|}^{2}-m_{W}^{2}-(2 k-1) \beta+\mathrm{i} \epsilon\right)}\right\} \\
& =p_{\|}^{\mu} \frac{p_{\perp}^{2}-z+2 n \beta}{4 p_{\perp}^{2} \sqrt{4 n \beta\left(n \beta-p_{\perp}^{2}-z\right)+\left(p_{\perp}^{2}-z\right)^{2}}} \theta\left[p_{\perp}^{2}-(\sqrt{z}+\sqrt{2 n \beta})^{2}\right] \theta[z],
\end{aligned}
$$

where $z=m_{W}^{2}+(2 k-1) \beta$. Here, we have neglected the electron mass $m_{e}$ as the smallest parameter, and the analysis has shown that this wouldn't cause any problems.

Combining the results of integration, one can obtain the resulting formula for the probability of the decay $v_{e} \rightarrow e^{-} W^{+}$:

$$
\begin{aligned}
w\left(v_{e} \rightarrow e^{-} W^{+}\right) & =\frac{G_{\mathrm{F}} m_{W}^{2} \beta}{\pi \sqrt{2} E} \sum_{k=0}^{k_{\max }} \sum_{n=0}^{n_{\max }(k)} \mathrm{e}^{-\chi} \sqrt{D^{-1}(\chi)}\{(\chi-\mu-k+1 / 2+n) \\
& \times\left[\frac{k !}{n !} \chi^{n-k}\left(L_{k}^{n-k}(\chi)\right)^{2}+\frac{(k-2) !}{(n-1) !} \chi^{n-k+1}\left(L_{k-2}^{n-k+1}(\chi)\right)^{2}\right] \\
& \left.-4(-1)^{k+n} \chi L_{k-1}^{n-k+1}(\chi) L_{n-1}^{k-n}(\chi)\right\} \theta[\sqrt{\chi}-\sqrt{\mu+k-1 / 2}-\sqrt{n}],
\end{aligned}
$$

where we use the following notations for the dimensionless variables: $\chi \equiv p_{\perp}^{2} /(2 \beta), \mu \equiv m_{W}^{2} /(2 \beta)$, and

$$
D(\chi)=(\chi-\mu-k+1 / 2+n)^{2}-4 n \chi .
$$


The theta-function in Eq. (12) allows to define the limits on summation over $n$ and $k$. Thus, for fixed $k$, the value $n$ should run from zero to its maximum, $n_{\text {max }}$ :

$$
n_{\text {max }}(k)=\text { IntegerPart }\left[(\sqrt{\chi}-\sqrt{\mu+k-1 / 2})^{2}\right],
$$

while the maximum value for $k$ is:

$$
k_{\text {max }}=\text { IntegerPart }[\chi-\mu+1 / 2] .
$$

As a consequence there are kinematically allowed Landau states for creating $W$ boson and electron originated from the threshold for neutrino energy.

Equation (12) should be compared with the asymptotic formula [8] obtained from the basic expressions for the electron and $W$ boson propagators in the Fock-Schwinger proper time formalism [24, 25] in the strong field limit $\left(p_{\perp}^{2} \gg m_{W}^{2} \gg e B \gg m_{e}^{2}\right)$ :

$$
w=\frac{G_{\mathrm{F}} \beta^{3 / 2} p_{\perp}}{\pi \sqrt{2 \pi} E} \Phi\left(\frac{4 \beta p_{\perp}^{2}}{m_{W}^{4}}\right)
$$

where

$$
\Phi(x)=\frac{1}{x} \int_{0}^{\infty} \frac{\mathrm{d} y}{y^{1 / 2}} \frac{(\sinh y)^{2}-y \tanh y}{(y-\tanh y)^{3 / 2}} \frac{(\tanh y)^{1 / 2}}{(\sinh y)^{2}} \exp \left[-\frac{y \tanh y}{x(y-\tanh y)}\right] .
$$

An independent evaluation of the process probability in a superstrong magnetic field was also derived within the instanton model [10].

The curves in Figs. 1, 2 display the probability $w(\chi)$ and the mean free path as functions of $\chi$. The monotone curves correspond to the asymptotic expression (16), while the "saw-tooth" profiles refer to the exact solution (12). Numerical calculation confirms that taking into account the contribution from only the ground Landau level, $n=0$, is insufficient in the context of the present task (see also Ref. [8]).

\section{Discussion}

The structure of the derived expression (12) indicates the existence of the abrupt increase of the probability (and decrease of the mean free path) at the energy values corresponding to the "turning on" the new Landau states. The exact expression affords to identify the quantum structure of the process. It is worth to note that the similar saw-tooth profile arises also in the problem of electron-positron pair creation by a photon in strong magnetic fields [26, 27]. As an example, in Ref. [26] the production of electron-positron pairs by single photons in magnetic fields of order $\geq 10^{12} \mathrm{G}$ was investigated in detail. It was shown that the attenuation coefficient (which is similar to the decay probability) exhibits a sawtooth pattern as a function of the photon energy, and its value is significantly greater at some energies than that predicted by the asymptotic expression.

The analysis of Eq. (12) affords to identify the similar tendency: for some specific values of parameters, the neutrino mean free path is significantly lower than it is predicted by the asymptotic formula. That corresponds to generation of a great amount of electrons and $W^{+}$bosons.

This fact could lead to several attractive conclusions. The subsequent $W^{+}$boson decay can influence the picture of the baryosynthesis in the Early Universe.

The Early Universe is known to be a natural laboratory for the processes in the extreme physical conditions. There are a number of bright ideas connecting cosmology and high energy physics. One of them is the domain structure of the Universe. 
The first papers which claimed the domain structure of vacuum as a consequence of CP-violation appeared in the 1970th. Thus, in Ref. [28] it was shown that while cooling the Universe the regions of two types should arise, divided by heavy domain walls that violate homogeneity. Domain structure became energeticaly not profitable so that every domain seeks to grow and absorb the others that leads to high velocities of domain walls [29]. The described processes could be extremely valuable for the generation of baryon asymmetry via anomalous electroweak processes (the so-called electroweak baryogenesis) in the models with thin or thick moving domain walls [30]. The important feature of such models is that the discussed energy region should be available at modern colliders.

In Ref. [31], the cosmological evolution of a universe filled with domains have been studied and it was concluded that at present the nearest wall should have gone beyond the horizon, leaving a trail in anisotropy in the black-body radiation [32].

Another attractive hypothesis claimed in Ref. [33] is that domain walls are ferromagnetic that allows to generate magnetic field strong enough to be a possible candidate of a primordial magnetic field leading to presently observable galactic fields. The idea was lately supported and discussed in Ref. [34-36], but received several contradict arguments [37, 38]. The authors of Ref. [39] claim that the submitted objections could only slightly decrease the effect but do not nullify it. It was suggested [35] that the effective surface tension of the domain walls can be made vanishingly small due to a peculiar magnetic condensation induced by fermion zero modes localized on the wall. As a consequence, the domain wall acquires a non-zero magnetic field perpendicular to the wall, and it becomes almost invisible as far as the gravitational effects are concerned.

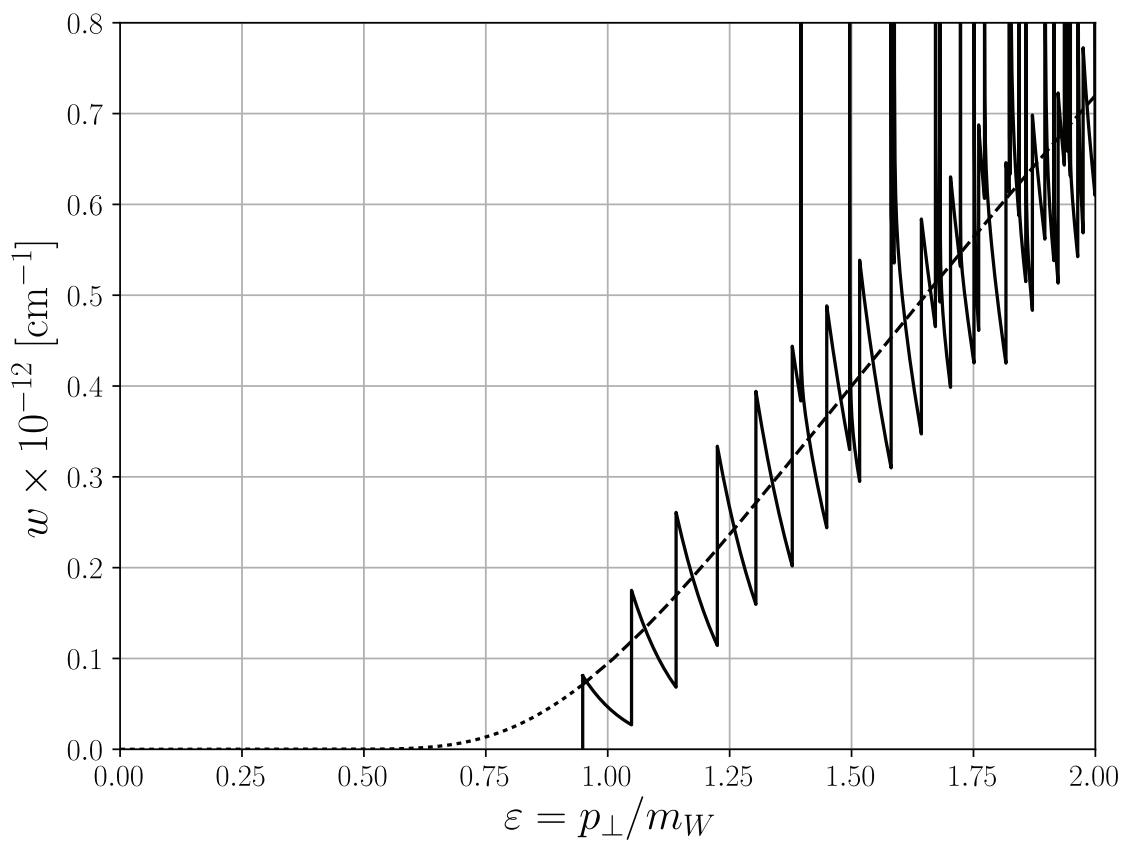

Figure 1. The decay $v_{e} \rightarrow e^{-} W^{+}$width for $\beta=0.1 m_{W}^{2}$ (the "sawtooth" profile). Dashed line: approximate formula without considering the quantizing effect of the magnetic field. 
It would be important that the process width (12) essentially depends on $p_{\perp}=E \sin \theta$, where $\theta$ is an angle between the neutrino momentum and the field direction. Suppose that the hypothesis of magnetic field generation in domain walls is correct. Then neutrinos moving almost perpendicular to the domain wall (i.e. almost parallel to the field direction) would fly it right through. On the other hand, neutrinos propagating almost parallel to the wall (in the way of the maximum magnetic field influence) with specific energies would predominantly decay, and would generate a great amount of $W$ bosons in the thickness of the wall. The subsequent decay of the $W$ boson by dominant quark channels could lead to some overabundance of hadron matter and antimatter inside domain walls to outside.

If the lepton-antilepton asymmetry, induced by the $C P$ violation in the lepton sector, has arisen before the electroweak phase transition, leading to overabundance of neutrinos over antineutrinos in the Universe, the considered mechanism would provide an overabundance of $W^{+}$and $e^{-}$over $W^{-}$and $e^{+}$inside domain walls. The $W^{+}$bosons would finally transform to protons while the $W^{-}$bosons would dominantly transform to antiprotons. The subsequent formation of the baryon asymmetry would paint the final picture of the Universe.

Of course these interesting applications should be studied precisely in the future taking into account the influence of other effects such as gravity.



Figure 2. The neutrino mean free path for $\beta=0.9 \mathrm{~m}_{W}^{2}$. Dashed line: approximate formula without considering the quantizing effect of the magnetic field. 


\section{Conclusion}

On the basis of the exact expressions of electron and $W$ boson propagators expanded over Landau levels, a neutrino decay in a superstrong magnetic fields (which may be characteristic for the Early Universe) was analysed.

It was shown that resonance peaks, observed in the probability of the process for specific neutrino momentum $p_{\perp}=E \sin \theta$, lead to electron and $W$ boson creation at specific Landau levels. It means that neutrino mean free path decreases sharply for certain values of $p_{\perp}$. The subsequent $W$ boson decay into the dominant quark channels should probably influence the matter composition within the domain walls.

There are still some open questions. Could the neutrino decay in a superstrong magnetic field really influence the matter composition within the domain walls in Early Universe? Would a possible $\mathrm{CP}$ violation in the lepton sector (Pontecorvo-Maki-Nakagawa-Sakata phase) lead to overabundance of neutrinos to antineutrinos? Could it lead, together with the CP violating phase in the CabibboKobayashi-Maskawa matrix, to any consequences? These will constitute the subject of further investigations.

\section{Acknowledgements}

The authors wish to thank D.A. Rumyantsev for useful discussions.

The research is financed by the grant of the Russian Science Foundation (Project No. 15-1210039).

\section{References}

[1] R. C. Duncan and C. Thompson, Astrophys. J. 392, L9 (1992)

[2] V. V. Skalozub, Phys. At. Nucl. 77, 901 (2014)

[3] L. M. Widrow et al., Space Sci. Rev. 166, 37 (2012)

[4] K. Subramanian, Rep. Prog. Phys. 79, 076901 (2016)

[5] A. V. Borisov, V. Ch. Zhukovskii, A. V. Kurilin and A. I. Ternov, Sov. J. Nucl. Phys. 41, 473 (1985) [Original Russian text: Yad. Fiz. 41, 743 (1985)]

[6] A. Erdas and M. Lissia, Phys. Rev. D 67, 033001 (2003)

[7] K. Bhattacharya and S. Sahu, Eur. Phys. J. C 62, 481 (2009)

[8] A. V. Kuznetsov, N. V. Mikheev and A. V. Serghienko, Phys. Lett. B 690, 386 (2010)

[9] A. A. Dobrynina and N. V. Mikheev, JETP 118, 54 (2014) [Original Russian text: Zh. Eksp. Teor. Fiz. 145, 65 (2014)]

[10] P. Satunin, JETP Letters 101, 657 (2014)

[11] T. Vachaspati, Phys. Lett. B 265, 258 (1991)

[12] J. Ambjørn and P. Olesen, Electroweak magnetism, W-codensation and antiscreening, in: Proc. of 4th Hellenic School on Elementary Particle Physics, (Corfu, 1992)

[13] D. Grasso and H. R. Rubinstein, Phys. Rep. 348, 163 (2001)

[14] V. I. Ritus, in Quantum Electrodynamics of Phenomena in an Intense Field (in Russian), Proc. P. N. Lebedev Physical Institute, 111, 5 (1979)

[15] C. Itzykson and J.-B. Zuber, Quantum Field Theory (McGraw-Hill, New York, 1985)

[16] V. O. Papanian and V. I. Ritus, in Issues in Intense-Field Quantum Electrodynamics, ed. by V. L. Ginzburg (Nova Science Publishers, New York), 153 (1989) 
[17] A. E. Shabad, in Polarization of the Vacuum and a Quantum Relativistic Gas in an External Field, ed. by V. L. Ginzburg (Nova Science Publishers, New York, 1992)

[18] I. M. Ternov, V. Ch. Zhukovskii and A. V. Borisov, Quantum Processes in Strong External Field, in Russian (Moscow State Univ., Moscow, 1989)

[19] A. V. Kuznetsov and N. V. Mikheev, Electroweak Processes in External Active Media (Berlin, Heidelberg, Springer-Verlag, 2013)

[20] A. Chodos, K. Everding and D. A. Owen, Phys. Rev. D 42, 288 (1990)

[21] A. V. Kuznetsov and A. A. Okrugin, Int. J. Mod. Phys. A 26, 2725 (2011)

[22] A. V. Kuznetsov, A. A. Okrugin and A. M. Shitova, Int. J. Mod. Phys. A 30, 1550140 (2015)

[23] A. A. Gvozdev and E. V. Osokina, Theor. Math. Phys. 170, 354 (2012) [Original Russian text: Teor. Mat. Fiz. 170, 423 (2012)]

[24] V. A. Fock, Phyzik. Z. Sowjetunion 12, 404 (1937)

[25] J. Schwinger, Phys. Rev. 82, 664 (1951)

[26] J. K. Daugherty and A. K. Harding, Astrophys. J. 273, 761 (1983)

[27] V. N. Baier and V. M. Katkov, Phys. Rev. D 75, 073009 (2007)

[28] I. Yu. Kobsarev, L. B. Okun and Ya. B. Zeldovich, Phys. Lett. B 50, 340 (1974)

[29] A. D. Dolgov and Ya. B. Zeldovich, Rev. Mod. Phys. 53, 1 (1981)

[30] D. S. Gorbunov and V. A. Rubakov, Introduction to the Theory of the Early Universe, Hot Big Bang Theory (World Scientific Publishing Company, Singapore, 2011)

[31] V. A. Kuzmin, V. A. Rubakov and M. E. Shaposhnikov, Phys. Lett. B 155, 36 (1985)

[32] L. B. Okun, Vacua, vacuum: the physics of nothing, History of Original Ideas and Basic Discoveries in Particle Physics (Plenum Press, New York, 1996)

[33] A. Iwazaki, Phys. Rev. D 56, 2435 (1997)

[34] P. Cea and L. Tedesco, Phys. Lett. B 425, 345 (1998)

[35] P. Cea and L. Tedesco, Phys. Lett. B 450, 61 (1999)

[36] M. M. Forbes and A. R. Zhitnitsky, Phys. Rev. Lett. 85, 5268 (2000)

[37] M. B. Voloshin, Phys. Lett. B 491, 311 (2000)

[38] M. B. Voloshin, Phys. Rev. D 63, 125012 (2001)

[39] L. Campanelli, P. Cea, G. L. Fogli and L. Tedesco, JCAP 0603, 005 (2006) 